\title{
ESTRATÉGIAS DE CONTROLE FÍSICO-MECÂNICO SOBRE Rumex obtusifolius
}

\author{
Germano Güttler ${ }^{1}$, Valmir Milani2 ${ }^{2}$ 'Camila Peligrinotti Tarouco ${ }^{3}$, Marcos André Nohatto ${ }^{4}$, Eliete de Fatima de \\ Ferreira da Rosa ${ }^{4}$ \\ ${ }^{1}$ Professor adjunto, Universidade do Estado de Santa Catarina, Lages, SC, CEP: 88520-000. \\ ${ }^{2}$ Engenheiro florestal, Universidade do Estado de Santa Catarina, Lages, SC, CEP: 88520-000. \\ ${ }^{3}$ Pós-doutoranda em Fisiologia Vegetal, Universidade Federal de Santa Maria, Santa Maria, RS, CEP: 97105-900. \\ ${ }^{4}$ Professor (a) adjunto, Instituto Federal Catarinense, Santa Rosa do Sul, SC, CEP: 88965-000
}

*Autor para correspondência: Camila Peligrinotti Tarouco, milatarouco@gmail.com

\begin{abstract}
RESUMO: A agricultura urbana tem crescido nos últimos anos decorrente das mudanças nos hábitos da população. Frente a isso, também evolui a necessidade de desenvolver sistemas de produção compatíveis com essa realidade. 0 objetivo do trabalho é avaliar os efeitos da capina, roçada e diferentes tipos de cobertura plástica sobre o controle da língua-de-vaca, uma das principais plantas daninhas difíceis de efetuar o controle na produção urbana. Para isso, foi desenvolvido experimento em delineamento experimental em blocos ao acaso, com cinco repetições. 0 experimento foi realizado em duas fases, sendo que a primeira foi constituída dos seguintes tratamentos: mulching preto, mulching preto com intermitência (aos 30 e 45 dias após a introdução do tratamento, o plástico foi removido temporariamente por 4 horas), mulching transparente, roçada, capina e testemunha; e na segunda fase, a área experimental foi mantida em pousio por 30 dias com intuito de verificar a capacidade de reinfestação da planta daninha. As variáveis avaliadas foram população de planta daninha e massa seca da parte aérea. Os resultados demonstram que especialmente a utilização do mulching apresenta-se com maior capacidade de reduzir a densidade populacional e massa seca de língua-de-vaca, porém a efetividade no controle depende da manutenção contínua no uso dessas estratégias.
\end{abstract}

PALAVRAS CHAVE: língua-de-vaca, planta daninha, agricultura urbana

\section{MECHANICAL-PHYSICAL CONTROL STRATEGIES FOR Rumex obtusifolius}

\begin{abstract}
Urban agriculture has grown in recent years due to changes in population habits. Faced with this, there is also a need to develop production systems compatible with this reality. The aim of this study is evaluate the effects of capture, mowing and different types of critical cover on the control of the bitter dock, one of the main weeds difficult to control urban production. For this, an experiment was developed in a randomized block design, with five replicates. Experiment performed in two phases, the first series consisted of treatments: black mulching, black mulching with intermittence (at 30 and 45 days after an introduction of the treatment, the plastic was removed temporarily for 4 hours), transparent mulching, mowing, weeding hoe and control treatment; and in the second phase, an experimental area to be kept in fallow for 30 days in order to verify the reinfestation capacity of the weed. The variables evaluated were weed population and shoot dry weight. The results demonstrate that especially the use of mulching has greater capacity to reduce the population density and shoot dry weight of bitter dock, but the effectiveness in the control depends on the continuous maintenance in the use of these strategies.
\end{abstract}

KEYWORDS: bitter dock; weed; urban agriculture

\section{INTRODUÇÃO}

A combinação entre agricultura e meio urbano, gera a impressão de incompatibilidade, porém sabese que essa é uma visão equivocada e limitada da realidade. Cada vez mais, percebe-se que a atividade urbana de produção de alimentos desempenha papel importante economicamente na geração de renda e sobrevivência das populações, além de fazer parte de políticas culturais e sociais. Com isso, é preciso buscar estratégias eficientes de produção que sejam viáveis e 
compatíveis com o ambiente da cidade.

Uma das principais dificuldades para produção agrícola nas cidades é o controle de plantas daninhas, uma vez que tais infestantes competem com a cultura pelos recursos de luz, água e nutrientes, prejudicando a qualidade e quantidade de produtos colhidos (Radosevich et al., 2007). Dentre as plantas daninhas relatadas na produção urbana, destaca-se a língua-devaca Rumex obtusifolius L. (Polygonaceae), capaz de se multiplicar através de sementes (60000 sementes planta $^{-1)}$ e propagação vegetativa por estruturas radiculares (Holm et al., 1977), o que amplia a dificuldade de controle da espécie.

A disponibilidade reduzida de herbicidas registrados para o controle da espécie (Agrofit, 2017), dificulta a utilização do método de controle químico, prejudicando o sistema produtivo. Com isso, gera-se a necessidade de trabalhar com outras alternativas de controle como a cobertura plástica do solo, roçada e capina, as quais baseiam-se na eliminação de plantas daninhas por meio de efeito físico-mecânico. Além do controle e menor impacto ambiental, tais estratégias são permitidas dentro da certificação orgânica (INSTRUÇÃO NORMATIVA Nº 007, DE 17 DE MAIO DE 1999), aumentando potencial de agregação do valor ao produto.

Nesse contexto, é fundamental avaliar a efetividade desses métodos de controle sobre plantas daninhas de difícil controle como a língua-de-vaca, bem como trabalhar no desenvolvimento da forma como tais técnicas serão implementadas na produção. Diante disso, este trabalho teve como objetivo avaliar os efeitos da capina, roçada e diferentes tipos de cobertura plástica (mulching) sobre o controle da língua-de-vaca (R. obtusifolius).

\section{MATERIAL E MÉTODOS}

0 experimento foi conduzido no período entre abril a agosto de 2016, em campo experimental localizado na área urbana de Lages-SC. 0 clima do local é do tipo Cfb, segundo a classificação de Köppen, com uma precipitação média anual de $1600 \mathrm{~mm}$. O solo no local do experimento é um Cambissolo Húmico. $O$ delineamento experimental utilizado foi blocos ao acaso, com cinco repetições. Cada parcela foi composta por área de $0,49 \mathrm{~m}^{2}(0,7 \times 0,7 \mathrm{~m})$, totalizando área total de $12,25 \mathrm{~m}^{2}$. A área útil utilizada para as avaliações foi de $0,25 \mathrm{~m}^{2}(0,5 \times 0,5 \mathrm{~m})$, desconsiderando as bordas restantes da parcela.

O preparo da área experimental foi efetuado por roçadeira mecânica rente ao solo previamente a implantação do experimento, não sendo efetuado a semeadura da língua de vaca, tampouco irrigação do local. Após esse procedimento, o experimento foi realizado em duas fases, sendo constituído dos seguintes tratamentos: mulching de polietileno preto, mulching preto com intermitência (aos 30 e 45 dias após a introdução do tratamento, o plástico foi removido temporariamente por 4 horas), mulching transparente, roçada mecânica das plantas invasoras rente ao solo, capina manual e testemunha. $\mathrm{Na}$ implantação do experimento e aos 60 dias após, foi realizada contagem da população de língua-de-vaca na área útil da parcela $(0,5 \times 0,5 \mathrm{~m})$. Na sequência, realizou-se a coleta dessas plantas na área amostrada para determinação da massa seca da parte aérea (MSPA). A quantificação da variável MSPA foi determinada pela pesagem das plantas após serem secas em estufa com circulação forçada de ar a $65^{\circ} \mathrm{C}$ por 72 horas.

A segunda fase do experimento iniciou após o término das avaliações da primeira fase, quando a área experimental foi mantida em pousio por 30 dias com intuito de verificar a capacidade de reinfestação da planta daninha. Durante esse período de pousio foram registradas temperaturas médias mensais para os meses de abril, maio, junho, junho e agosto $(12,6,8,9,11,1$ e $12,6^{\circ} \mathrm{C}$ respectivamente). A precipitação acumulada durante os meses de condução do experimento foi de $428,3 \mathrm{~mm}$ (INMET, 2016). Foi realizado a contagem da população da planta daninha em estudo (língua de vaca) ao final do experimento e determinação da MSPA, conforme descrito anteriormente.

Os dados coletados foram submetidos à análise de variância pelo teste F. Quando constatada significância estatística, procedeu-se à comparação entre as médias utilizando o teste de Duncan $(p \leq 0,05)$ no programa Assistat $\AA$ (Silva e Azevedo, 2009).

\section{RESULTADOS E DISCUSSÃO}

Com relação à primeira fase do experimento, observa-se diferença entre os tratamentos para a variável densidade populacional quantificada aos 60 dias após implantação do experimento e MSPA. Os resultados indicam que a utilização do mulching, 
especialmente de forma contínua, nessa primeira fase avaliada, foi efetivo na redução da população da planta daninha (em termos percentuais, redução de $87 \%$ para o mulching contínuo) (Tabela 1), além de suprimir o desenvolvimento da espécie, demonstrado pela avaliação da MSPA, onde verificou-se menor valor numérico da variável comparada aos outros tratamentos (Tabela 2). Entre os tratamentos com utilização de mulching, o transparente foi o menos efetivo na primeira fase, apresentando redução da população de apenas $24,7 \%$ (Tabela 1).

Tabela 1. População de plantas de língua-de-vaca (Rumex obtusifolius) (número corresponde à média de indivíduos obtida nas cinco unidades experimentais de $0,25 \mathrm{~m}^{2}$ por tratamento) em resposta a diferentes estratégias de controle físico-mecânico.

\begin{tabular}{lccc}
\hline \multirow{2}{*}{ Tratamento } & \multicolumn{2}{c}{ Primeira fase } & Segunda fase \\
\cline { 2 - 4 } & População inicial & População aos 60 DAT $^{1}$ & População reinfestante aos 90 DAT $^{2}$ \\
\hline Mulching contínuo & $171,4^{\text {NS }}$ & $21,0 \mathrm{~d}^{3}$ & $469,5^{\text {NS }}$ \\
\hline Mulching intermitente & 153,8 & $65,4 \mathrm{~cd}$ & 351,5 \\
\hline Mulching transparente & 176,6 & $133,0 \mathrm{bc}$ & 408,7 \\
\hline Capina & 117,0 & $476,6 \mathrm{a}$ & 605,3 \\
\hline Roçada & 160,2 & $199,6 \mathrm{~b}$ & 281,4 \\
\hline Testemunha & 231,4 & $222,0 \mathrm{~b}$ & 401,5 \\
\hline CV (\%) & 42,2 & 41,9 & 58,2 \\
\hline
\end{tabular}

${ }_{1}^{1}$ População de língua-de-vaca avaliada aos 60 dias após implantação do experimento;

${ }^{2}$ População de língua-de-vaca avaliada 90 DAT, considerando 30 dias da área em pousio;

${ }^{3}$ Médias seguidas por letras minúsculas diferentes na coluna diferem estatisticamente através do teste Duncan $(p \leq 0,05)$. NS Não significativo.

Tabela 2. Massa seca da parte aérea (MSPA) de língua-de-vaca (Rumex obtusifolius) (gramas corresponde à média obtida nas cinco unidades experimentais de $0,25 \mathrm{~m}^{2}$ por tratamento) em resposta a diferentes estratégias de controle físico-mecânico.

\begin{tabular}{lcc}
\hline \multirow{2}{*}{ Tratamento } & \multicolumn{1}{c}{ Primeira fase } & Segunda fase \\
\cline { 2 - 3 } & Massa seca da parte aérea aos 60 DAT & Massa seca da parte aérea reinfestante aos 90 DAT ${ }^{2}$ \\
\hline Mulching contínuo & $0,4 \mathrm{~b}$ & $3,8 \mathrm{c}$ \\
\hline Mulching intermitente & $1,2 \mathrm{~b}$ & $10,1 \mathrm{bc}$ \\
\hline Mulching transparente & $9,2 \mathrm{~b}$ & $8,5 \mathrm{bc}$ \\
\hline Capina & $19,3 \mathrm{~b}$ & $17,0 \mathrm{~b}$ \\
\hline Roçada & $25,6 \mathrm{~b}$ & $15,4 \mathrm{bc}$ \\
\hline Testemunha & $74,8 \mathrm{a}$ & $32,9 \mathrm{a}$ \\
\hline CV $(\%)$ & 37,1 & 60,5 \\
\hline
\end{tabular}

${ }^{1}$ Massa seca da parte aérea (gramas) de língua-de-vaca avaliada aos 60 dias após implantação do experimento;

${ }^{2}$ Massa seca da parte aérea (gramas) de língua-de-vaca avaliada 90 DAT, considerando 30 dias da área em pousio;

${ }^{3}$ Médias seguidas por letras minúsculas diferentes na coluna diferem estatisticamente através do teste Duncan $(p \leq 0,05)$.

O efeito negativo do mulching contínuo sobre os propágulos de plantas daninhas foi demonstrado por Ashrafuzzaman et al. (2011), que avaliou diferentes cores de cobertura (preto, transparente e azul), resultando na redução da quantidade de plantas daninhas $\mathrm{m}^{-2}$, especialmente no mulching preto, semelhante ao que ocorreu no experimento. Apesar de não ter sido avaliado a intensidade luminosa nos tratamentos, acredita-se que o mulching preto apresenta maior capacidade de bloqueio da entrada da luz, o que prejudicou a emergência da língua-de-vaca.

Além do efeito físico, hipotetiza-se que mesmo com uso de cobertura plástica, ainda houve predominância de temperaturas baixas ao longo do experimento. Estudo com objetivo de determinar a influência da luz e da temperatura sobre a germinação de 
sementes da língua-de-vaca conduzido por Benvenuti et al. (2000), demonstrou que sob temperatura de 10$15^{\circ} \mathrm{C}$ ocorre menor germinação no escuro comparado a luz, que ajuda explicar em parte os menores valores observados no tratamento de mulching preto contínuo em relação ao mulching transparente.

A utilização do mulching como cobertura de solo reduz as perdas de umidade por evaporação, aumentando a eficiência da utilização da água (Zhang et al., 2011); elevação na temperatura na camada superficial do solo, o que pode favorecer a atividade radicular especialmente em regiões mais frias (DíazPérez, 2009) e acelerar a decomposição da matéria orgânica, incrementando a disponibilidade de nutrientes para as plantas (Stapleton, 2000).

Para a variável densidade populacional, também destaca-se que o tratamento com uso da capina apresentou maior número de indivíduos (Tabela 1). O corte da planta rente ao solo manteve estruturas subterrâneas da planta, possivelmente dotadas de grande quantidade de gemas, reservas e fitohormônios como as citocininas, importantes estimulantes da divisão das células, indução e proliferação de novas brotações (Howell et al., 2003). Apesar disso, a superioridade observada na população de plantas do tratamento capina em relação aos demais tratamentos não se manteve para MSPA (Tabela 2). Isso deve-se ao tamanho diminuto das plantas (tal percepção foi feita de forma visual), que no montante total apresentaram pouca influência na definição da MSPA.

A capacidade da planta daninha apresentar mecanismos alternativos de reprodução proporciona vantagens na sobrevivência da espécie (Kissmann e Groth, 1992). Algumas espécies de plantas daninhas como a língua-de-vaca apresentam tanto reprodução seminifera (sementes) quanto reprodução vegetativa por partes radiculares, dificultando a eficiência das estratégias de controle (Kissmann e Groth, 1992).

A determinação da variável MSPA demonstra que todos os tratamentos testados para o controle apresentam menores valores em comparação a testemunha (Tabela 2), ou seja, independentemente da população resultante pós efeito dos tratamentos e nível de eficiência, verifica-se que a utilização das estratégias de manejo (mulching, capina e roçada) ainda posicionam-se como melhor alternativa do que o pousio.

$\mathrm{Na}$ segunda fase experimental, apesar da grande variação numérica para a densidade não constatou-se diferença estatística entre os tratamentos, ou seja, sob condição de pousio, a espécie volta a predominar no ambiente, inclusive, os dados demonstram uma evolução nos valores em relação a população detectada na primeira fase do experimento (Tabela 1).

Apesar dessa reinfestação da língua-de-vaca nas unidades experimentais, essas apresentaram menores valores de MSPA comparado a testemunha (Tabela 2), indicando possível efeito residual das estratégias de controle realizadas na primeira fase do experimento. Estudo desenvolvido por Ricci et al. (2000) que avaliou os efeitos da solarização, proporcionada por cobertura plástica, sobre a reinfestação de tiririca (Cyperus rotundus) demonstrou que após a remoção do mulching houve redução da população da planta daninha, onde as parcelas não solarizadas alcançaram 1.151 plantas $\mathrm{m}^{-2}$, contra 473 plantas $\mathrm{m}^{-2}$ nas solarizadas. Além disso, o estudo citado mostrou incremento $28 \%$, $34 \%$ e $37 \%$ de na produtividade de cenoura, repolho e beterraba, respectivamente. 0 potencial de controle baseia-se numa técnica conhecida como solarização do solo, que aproveita a energia solar para desinfestação do solo com uso de mulching (Ghini, 1997).

Os resultados demonstram que especialmente a utilização do mulching apresenta-se com maior capacidade de reduzir a densidade populacional e massa seca de língua-de-vaca, porém a efetividade no controle depende da manutenção contínua no uso dessa estratégia.

\section{REFERÊNCIAS BIBLIOGRÁFICAS}

AGROFIT. Sistemas de agrotóxicos fitossanitários. Disponível em: http://agrofit.agricultura.gov.br/agrofit_ cons/principal_agrofit_cons. Acesso: out 2017.

Ashrafuzzaman, M.; Halim, M.A.; Ismail, M.R.; Shahidullah, S.M.; Hossain, M.A. Effect of plastic mulch on growth and yield of chilli (Capsicum annuum L.). Brazilian Archives of Biology and Technology. 2011, 54, 2, 321-330.

Benvenuti, S.; Macchia, M.; Miele, S. Light, temperature and burial depth effects on Rumex obtusifolius seed germination and emergence. Weed Research. 2001, 41, 2, 177-186. 
Díaz-Perez, J.C. Root zone temperature, plant growth and yield of broccoli [Brassica oleracea (Plenck) var. italica] as affected by plastic film mulches. Scientia Horticulturae. 2009, 123, 2, 156-163.

Ghini, R. Desinfestação do solo com uso de energia solar: solarização e coletor solar. Jaguariúna: Embrapa - CNPMA. 1997. 29p. (Embrapa - CNPMA, Circular, 1)

Holm, L.G.; Plucknett, D.L.; Pancho, J.V.; Herberger, J.P. Rumex crispus and obtusifolius. In. The World's worst weeds: distribution and biology. University Press of Hawaii. Honolulu, USA. 1977.

Howell, S.H.; Lall, S.; Che, P. Cytokinins and shoot development. Trends in Plant Science. 2003, 8, 9, 453459.

Kissmann, K.G.; GROTH, D. Plantas infestantes e nocivas. São Paulo: Basf Brasileira, 1992. 789 p.

INMET. Instituto Nacional de Meteorologia. Tempo. Disponível em: http://www.inmet.gov.br/portal/. Acesso: mar 2018.

INSTRUÇÃO NORMATIVA N 007, DE 17 DE MAIO DE 1999. Disponível em: http://ibd.com.br/Media/arquivo digital/c40fe6c4-51f3-414a-9936-49ea814fd64c.pdf. Acesso: out 2017.
Radosevich, S.R.; Holt, J.S.; Ghersa, C.M. Ecology of weeds and invasive plants: Relationship to agriculture and natural resource management. 3.ed. Hoboken: John Wiley \& Sons, 2007. 479p.

Ricci, M.S.F.; Almeida, D.L.A.; Fernandes, M.C.A.; Ribeiro, R.L.D.; Cantanheide, M.C.S. Efeitos da solarização do solo na densidade populacional da tiririca e na produtividade de hortaliças sob manejo orgânico. Pesquisa Agropecuária Brasileira. 2000, 35, 11, 2175-2179.

Silva, F.A.S.; Azevedo, C.A.V. Principal Components Analysis in the Software Assistat-Statistical Attendance. In: WORLD CONGRESS ON COMPUTERS IN AGRICULTURE, 7, Reno-NV-USA: American Society of Agricultural and Biological Engineers, 2009.

Stapleton, J.J. Soil solarization in various agricultural production systems. Crop Protection. 2000, 19, 8, 837841.

Zhang, S.; LI, P.; Yang, X.; Wang, Z.; Chen, X. Effects of tillage and plastic mulch on soil water, growth and yield of spring-sown maize. Soil and Tillage Research. 2011, $112,1,92-97$. 